\title{
A Two-stage Cardiac PET and Late Gadolinium Enhancement MRI Co-registration Method for Improved Assessment of Non-ischemic Cardiomyopathies Using Integrated PET/MR
}

\author{
Zheng Zhang \\ Shanghai Jiao Tong University \\ Xing Chen \\ Shanghai East Hospital \\ Qing Wan \\ Shanghai East Hospital \\ Haiyan Wang \\ Shanghai East Hospital
}

$\mathrm{Na}$ Qi

Shanghai East Hospital

Zhiwen You

Shanghai East Hospital

Jianmin Yuan

United Imaging Healthcare Co.Ltd

Lingzhi Hu

United Imaging Healthcare Co. Ltd

Hongwei Sun

United Imaging Healthcare Co, Ltd

Zhe Wang

United Imaging Healthcare Co.Ltd

Chenxi Hu

Shanghai Jiao Tong University

Jun Zhao ( $\nabla$ petcenter@126.com )

Shanghai East Hospital https://orcid.org/0000-0002-9887-5512

\section{Research Article}

Keywords: cardiac PET/MR, image co-registration, non-ischemic cardiomyopathy, Late Gadolinium Enhancement Image, 18F-FDG PET 
Posted Date: December 23rd, 2021

DOl: https://doi.org/10.21203/rs.3.rs-978627/v1

License: (c) (i) This work is licensed under a Creative Commons Attribution 4.0 International License. Read Full License

Version of Record: A version of this preprint was published at European Journal of Nuclear Medicine and Molecular Imaging on January 15th, 2022. See the published version at https://doi.org/10.1007/s00259022-05681-4. 


\section{Abstract}

\section{Purpose}

Respiratory motion causes mismatches between PET images of the myocardium and the corresponding cardiac MR images in cardiac integrated PET/MR. The mismatch may affect the attenuation correction and the diagnosis of non-ischemic cardiomyopathies. In this study, we present a two-stage cardiac PET and MR Late Gadolinium Enhancement (LGE) co-registration method, which seeks to improve diagnostic accuracy of non-ischemic cardiomyopathies via better image co-registration using an integrated wholebody PET/MR system.

\section{Methods}

The proposed PET and LGE two-stage co-registration method was evaluated through comparison with one-stage direct co-registration and no-registration. One hundred and ninety-one slices of LGE and forty lesions were studied. Two trained nuclear medicine physicians independently assessed the displacement between LGE and PET to qualitatively evaluate the co-registration quality. The changes of the mean SUV in the normal myocardium and the LGE-enhanced lesions before and after image co-registration were measured to quantitatively evaluate the accuracy and value of image co-registration.

\section{Results}

The two-stage method had an improved image registration score $(4.93 \pm 0.89)$ compared with the noregistration method $(3.49 \pm 0.84, p$ value $<0.001)$ and the single-stage method $(4.23 \pm 0.81, p$ value $<0.001)$. Furthermore, the two-stage method led to increased SUV value in the myocardium (3.87 \pm 2.56$)$ compared with the no-registration method $(3.14 \pm 1.92, p$ value $<0.001)$ and the single-stage method $(3.32 \pm 2.16, p$ value $<0.001$ ). The mean SUV in the LGE lesion significantly increased from $2.51 \pm 2.09$ to $2.85 \pm 2.35$ ( $p$ value $<0.001)$ after the two-stage co-registration.

\section{Conclusion}

The proposed two-stage registration method significantly improved the co-registration between PET and LGE in integrated PET/MR imaging. The technique may improve diagnostic accuracy of non-ischemic cardiomyopathies via better image co-registration.

Registered No.

DF-2020-085, 2020.04.30

\section{Introduction}

Positron emission tomography (PET) and magnetic resonance imaging (MRI) have been widely used for the investigation of cardiovascular disease for decades. With the introduction of integrated PET/MR in 
2010, PET/MR shows high potential for innovation in cardiac imaging [1]. Late gadolinium enhancement (LGE) on MRI can detect focal fibrosis in myocardium and provide excellent risk stratification for sudden cardiac death, all-cause mortality, and heart failure hospitalization in patients with non-ischemic cardiomyopathy [2]. The combination of metabolic information from ${ }^{18} \mathrm{~F}$-fluoro-deoxy-glucose positron emission tomography ( ${ }^{18} \mathrm{~F}-\mathrm{FDG}$ PET) and LGE may provide supplementary evidence for the evaluation of myocardial vitality and inflammation of dilated cardiomyopathy (DCM) or hypertrophic cardiomyopathy (HCM).

However, co-registration between MRI and PET of heart is challenging due to patient involuntary motion including respiration and cardiac contraction [3]. Mäkela et al. summarized the co-registration accuracy for intra- and intermodal cardiac applications and led to a conclusion that co-registration of any data not acquired simultaneously is a major problem in inter- and intra-modal examinations [4]. However, image misalignment can still exist in integrated PET/MR imaging due to different acquisition schemes of two modalities, although MRI and PET data are acquired simultaneously.

While misregistration between PET and LGE is an important confounder in accurate assessment of nonischemic cardiomyopathies with integrated PET/MR, the techniques developed for co-registration of PET and LGE in integrated PET/MR remain under developed. For cardiac PET/CT imaging, respiratory motion is believed to lead to significant bias in myocardial uptake of FDG $[5,6]$. A robust registration approach in clinical practice may be to correct for the mismatch caused by respiratory motion while ignoring other factors [7]. Strategies used in PET/CT to correct the respiratory motion introduced myocardial mismatches can also be used in PET/MR. It starts from aligning single-phase CT to respiratory-gated PET [8], phase-matched PET and CT in 4D-PET/CT imaging [9], and shallow tidal free-breathing or endexpiratory breath-hold CT aligned to free-breathing PET in clinical practice [10].

In this study, we present a two-stage cardiac PET/LGE co-registration method to improve the assessment of non-ischemic cardiomyopathies with integrated PET/MR. We hypothesize that respiratory motion is the main cause of the misregistration between LGE and PET for cardiac imaging. The mismatch between LGE and PET can be eliminated by using a two-step approach to resolve the mismatch caused by respiratory movement. Evaluation of the registration quality was performed based on clinical patients using a set of qualitative and quantitative criteria.

\section{Materials And Methods}

Study population

A total of 33 patients referred to cardiac ${ }^{18}$ F-FDG PET/MR scans between May 2020 and September 2021 were retrospectively enrolled into the study. All patients had documented non-ischemic cardiomyopathies ( $\mathrm{HCM}$ or DCM) without contraindications against MR or PET imaging. Of the 33 patients, one patient was excluded because of incomplete data acquisition, three excluded because of severe motion artifacts in the LGE images, and one excluded because of failed attenuation correction, potentially due to the 
presence of strong body motion. The final cohort consisted of 28 patients, including fourteen DCM and five HCM patients were evaluated for viability assessment with the patient preparation of glucose loading, four DCM and six HCM patients were evaluated for detecting potential inflammation with the patient preparation of fasting. The patient characteristics and datasets, described in detail in Table 1. This is a post hoc analysis of a previous prospective study conducted at Shanghai East Hospital, Tongji University (Registered No. DF-2020-085; primary aim: to evaluate the value of ${ }^{18} \mathrm{~F}-\mathrm{FDG}$ PET/MR imaging in cardiovascular disease. All patients signed informed consent forms).

Integrated PET/MR imaging

All patients underwent ${ }^{18} \mathrm{~F}$-FDG PET/MR examination using a United Imaging uPMR790 scanner (United Imaging Healthcare Co. Ltd., Shanghai, China) [11]. For patients undergoing myocardial viability assessment, a patient preparation protocol of glucose loading and insulin administration was carried out after a minimal fasting period of six hours [12]. For patients undergoing myocardial inflammation assessment, patients were instructed to take a diet rich in fats with no carbohydrates and then fasted for 12 hours before the exam [13]. Both the patient preparation procedures are followed by the intravenous administration of ${ }^{18} \mathrm{~F}-\mathrm{FDG}(222-296 \mathrm{MBq})$.

List-mode PET imaging was performed at a median uptake time of 60 minutes. The imaging typically lasted 40 minutes. The data were reconstructed using an Ordered Subsets Expectation Maximization iterative reconstruction algorithm (OSEM) with two iterations, 20 subsets and matrix size $150 \times 150$. Images were spatially smoothed with $3 \mathrm{~mm}$ full-width at half-maximum (FWHM) gaussian kernels. PET emission data were corrected for scatter, random, dead time, and attenuation. Two PET datasets were reconstructed from each examination, either with no respiratory gating (termed ungated PET) or eightphase respiratory gating (termed respiratory-phase-resolved PET) based on concurrently acquired respiratory bellows signal (Invivio Expression, Gainesville, FL). The latter amounts for a five-minute acquisition for each respiratory phase, which was believed to generate an acceptable quality. The PET image was acquired with concurrent recording of the respiratory bellows signal. The same bellows signal was used to gate the acquisition of the FLASH image, which was acquired with free breathing instead of breath-hold. The PET image was then reconstructed by using only the data collected in the end-expiration phase, which was the same phase used for gating the FLASH image acquisition.

A segmented MR-based attenuation correction (MRAC) map for ungated PET and phase-resolved PET was generated by using the same respiration-gated Dixon 3D fast low angle shot (FLASH) sequence [14]. Notice that the FLASH sequence was also used as an intermedia for improved PET/LGE co-registration, as described below. The FLASH sequence was acquired with patient free-breathing, and retrospective resorting of the data based on concurrently acquired respiratory bellows signal was performed to generate reconstruction at end expiration only. The imaging parameters of the Dixon 3D FLASH were: field of view $500 \times 350 \mathrm{~mm}$, matrix $493 \times 384$, acquired resolution, $0.91 \times 0.91 \times 2 \mathrm{~mm}^{3}$; repetition time, 4.91 milliseconds; echo time, 2.24/3.19 milliseconds; and flip angle, 10 degrees, acquisition time, 150 seconds. 
MR imaging was performed with a 12-channel phased-array body surface coil and a 24-channel phasedarray spine coil. The imaging protocol includes chronologically a whole-heart morphological imaging, a multi-slice whole-heart cine imaging, and a multi-slice whole-heart short-axial Late Gadolinium Enhancement (LGE) imaging. For LGE, the Phase-Sensitive Inversion-Recovery prepared T1-weighted gradient-echo pulse sequence (PSIR) was acquired 10 minutes after the intravenous administration of 0.3 mmol gadopentetate dimeglumine (Magnevist; Bayer HealthCare, Wayne, NJ) per kilogram. All LGE images were obtained during an end-expiratory breath-hold.

\section{A two-stage PET/LGE co-registration method}

A two-stage registration method was developed to accurately register the 3D PET images with the 2D multi-slice MR LGE images. The schematic of the registration method is shown in Fig 1A. In the first step, each 3D respiratory-phase resolved PET image was compared to the 3D end-respiratory MRAC FLASH image to identify the PET image best matched to the MRAC FLASH image. The major landmark features for selecting the best PET image included the overall shape of the heart and its interface with the lung and liver. These features were chosen because they were relatively stable regardless of the patient preparation methods. Typically, the same respiratory phase of PET was found in each patient, corresponding to the phase employed by MRAC reconstruction, since the same bellows signal was used. In the second step, a rigid registration was executed to register each slice of the 2D LGE images with the 3D FLASH image to generate a 3D displacement field that captures the perceived motion from the 3D FLASH to each 2D LGE image. Manual registration was performed in superior-inferior (S-I) direction and in-plane successively on uWS-MR workstation (United Imaging Healthcare Co. Ltd., Shanghai, China). Inner landmarks of myocardium (papillary muscles, the insertion point of, the right ventricle into the septum, the most inferior aspect of the septum, and the most inferior aspect of the lateral wall) were used for the rigid registration [4]. Finally, the 3D displacement field was used to warp the FLASH-registered PET, resulting in a 2D PET image co-registered with each 2D LGE image. The displacement in superior-inferior direction was recorded to assess the mismatch caused by respiratory movement.

Myocardial lesion identification

The location and boundary of myocardial lesions on LGE images were identified together by a nuclear medicine physician with three years' experience in cardiac PET/MR imaging and a cardiologist with five years' experience on MR imaging. A manual computing tool (closed polygon) was used to create the region of interest (ROI) on LGE image. Since LGE and PET share the same patient coordinate system in integrated PET/MR, the ROI can be directly mapped to PET image to measure SUV value of the lesion.

\section{SUV measurement of myocardium}

SUV value of myocardium was measured before and after registration correction. After loading the LGE and PET images into the Fusion software of the workstation, a manual computing tool (circular or closed polygon) was used to delineate the outer and inner boundaries of the myocardium in the LGE image, 
resulting in two ROIs mapped to the PET image, termed Myo-outer and Myo-inner. Area ${ }^{160-o u t e r}$ and Area ${ }^{260-i n s e r}$ are the area of Myo-outer and Myo-inner. Myocardium SUV $\operatorname{men}$ was calculated according to:+

Myocardium SUV mean

$=\left(S U V_{\text {mean }}^{\text {Myo-outer }} \times\right.$ Area $^{\text {Myo-outer }}-S U V_{\text {mean }}^{\text {Myo-inner }} \times$ Area $\left.^{\text {Myo-inner }}\right) /\left(\right.$ Area $^{\text {Myo-outer }}-$ Area $\left.^{\text {Myo-inner }}\right)$

Image analysis

The performance of the proposed two-stage PET/LGE co-registration method was evaluated through comparison with no-registration and one-stage direct PET/LGE co-registration, using an image analysis process shown in Fig 2. Three sets of PET images, labeled in Fig 2 as PET image1/2/3, were generated from this process. PET image1 (ungated PET) was reconstructed based on all PET raw data. No explicit co-registration was performed to generate PET image1 except for the intrinsic motion robustness encompassed by the integrated PET/MR imaging. PET image2 (single-phase gated PET) was a direct coregistration between the respiratory-phase resolved PET images and each of the multi-slice 2D LGE images (termed one-stage registration). The co-registration was manually performed by selecting the PET image best matched to each of the 2D LGE slice. The one-stage method is illustrated in Fig 1B. PET image3 was generated based on the proposed method.

Evaluation of the registration quality from each of the three methods was performed based on a set of qualitative and quantitative criteria. For qualitative evaluation, two trained nuclear medicine physicians independently assessed the displacement between LGE and PET for each method. Using normal left ventricular myocardial thickness (LV. Thk) as a reference, a registration accuracy score ranging from 1 to 5 was rated for each method. Score definition: 1 point, PET/LGE Displacement $\geq$ Twofold LV.Thk; 2

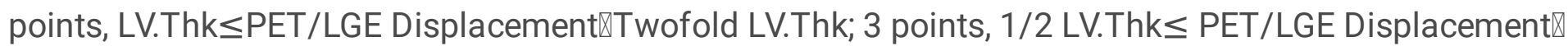
LV.Thk; 4 points, PET/LGE Displacement $\leq 1 / 2$ LV.Thk; 5 points, undetectable mismatches between images. For patients with low myocardial FDG uptake, the overall shape of the heart and its border with the lung and liver were still visible, which were used for scoring. The analysis was performed in all 28 patients for each co-registration method. The scores from two reviewers were averaged before further comparison.

For quantitative evaluation, the changes of the SUVmean before and after image co-registration were measured in the normal myocardium (typically three slices were selected by an experienced nuclear technologist per patient: basal, middle, apex) for each co-registration method, using the method described in the previous subsection. Since SUVmean qualifies as a surrogate for co-registration accuracy only for patients with higher FDG uptake in the myocardium than blood, the comparison between different coregistration methods was firstly performed within these patients. However, since different patient preparation methods (glucose-loading vs fasting) led to different PET image appearances, the same comparison was also performed separately for each of the glucose-leading $(n=18)$ and fasting $(n=10)$ 
subgroups. Finally, SUVmean in the LGE lesion was evaluated to reflect the impact of co-registration on assessment of non-ischemic cardiomyopathies in cardiac integrated PET/MR.

Statistical analysis

Wilcoxon signed-rank test and paired t-test were performed for evaluating statistical differences in the qualitative scores and the mean SUVs, respectively, between each pair of co-registration methods.

Table 1 Patient characteristics and datasets

\begin{tabular}{lll} 
& All patients & Patients with PLRA \\
\hline N & 33 & 28 \\
\hline Age (years) & $56 \pm 17$ & $57 \pm 18$ \\
\hline Male (n) & 24 & 21 \\
\hline Female (n) & 9 & 7 \\
\hline Incomplete data acquisition & 1 & 0 \\
\hline Severe motion artifacts in LGE & 3 & 0 \\
\hline HCM (n) & 12 & 11 \\
\hline HCM with Fasting & 7 & 6 \\
\hline HCM with GL & 5 & 5 \\
\hline HCM with Low myocardial glucose uptake & 0 & 0 \\
\hline DCM (n) & 21 & 17 \\
\hline DCM with Fasting & 6 & 4 \\
\hline DCM with GL & 15 & 13 \\
\hline DCM with Low myocardial glucose uptake & 4 & 0
\end{tabular}

PLRA PET and LGE Registration Assessment, GL Glucose Loading

\section{Results}

Of the 28 patients included for further analysis in the study, 25 showed higher myocardial FDG uptake than the blood pool. Three patients in the fasting group had myocardial FDG uptake lower than or equal to the blood pool. The registration quality assessment included 191 slices of LGE for qualitative evaluation, 76 slices of normal myocardium and 40 slices of LGE-enhanced myocardium LGE for quantitative evaluation.191 LGE/PET displacement data in S-I direction were recorded. To validate that the PET and MRAC were intrinsically co-registered, an experienced reader was asked to perform 
comparison of the two images in the set of patients with higher myocardial FDG uptake than the blood pool $(n=26)$. It was found that the co-registration was accurate in 25 of 26 patients, with only one patient as an exception, where we suspected that the cause of misregistration was patient motion. For patients with low myocardial FDG uptake, we did not perform extra comparison between the two images and directly used the end-expiratory PET image. Although patient motion may be a confounder to the coregistration quality in these patients, the previous analysis showed such a risk is quite low.

The first step in two-stage LGE/PET image co-registration is to select a single-phase PET best matched to the FLASH from respiratory-phase resolved PET images. Compared with the ungated PET (PET image1), the FLASH matched single-phase PET image showed better registration quality. The registration score increased from $4.375 \pm 0.625$ to $4.813 \pm 0.410$. The increase in registration quality was statistically significant $(p$ value $<0.001)$.

The qualitatively and quantitatively analysis results over all patients with higher FDG uptake in the myocardium than blood are shown in Fig 3. For qualitative evaluation, PET image3 showed the best image registration score $(4.93 \pm 0.89)$ compared with PET image1 $(3.49 \pm 0.84$, p value $<0.001)$ and PET image2 (4.23 \pm 0.81 , $p$ value $<0.001$ ), and also PET image2 was better than PET image1 ( $p$ value $<0.001$ ). For quantitative evaluation, PET image3 had the highest SUV value of the normal myocardium (3.87 \pm 2.56$)$ compared with PET image1 $(3.14 \pm 1.92$, $p$ value $<0.001)$ and PET image2 $(3.32 \pm 2.16$, $p$ value $<0.001$ ), and also PET image2 was slightly better than PET image1 ( $p$ value $=0.0012$ ).

The subgroup analysis is shown in Fig 4. For qualitative evaluation of glucose loading patients, PET image3 showed the best image registration score $(4.96 \pm 0.16)$ compared with PET image1 $(3.69 \pm 0.97, \mathrm{p}$ value $<0.001)$ and PET image2 $(4.28 \pm 0.83, p$ value $<0.001)$, and also PET image2 was better than PET image1 ( $p$ value $<0.001$ ). For qualitative evaluation of fasting patients, PET image3 showed the best image registration score $(4.90 \pm 0.24)$ compared with PET image1 $(3.69 \pm 0.92$, $p$ value $<0.001)$ and PET image2 $(4.44 \pm 0.76, p$ value $<0.001)$, and also PET image2 was better than PET image1 ( $p$ value $<0.001)$. The trends are similar in regard to the qualitative analysis between the two subgroups. For quantitative evaluation of glucose loading patients, PET image3 had the highest SUV value of myocardium (4.37 \pm 2.85$)$ compared with PET image1 $(3.52 \pm 2.15, p$ value $<0.001)$ and PET image2 $(3.78 \pm 2.45, p$ value $<0.001$ ), and also PET image 2 was slightly better than PET image 1 ( $p$ value $=0.0011$ ). For quantitative evaluation of fasting patients, PET image3 had a trending increase of SUV value of myocardium $(1.96 \pm 1.27)$ over PET image1 $(1.77 \pm 1.01, p$ value $=0.056)$ and a marginal increase over PET image2 $(1.81 \pm 1.06, p$ value $=0.033$ ). PET image2 was slightly better than PET image1 ( $p$ value $=0.4475)$. The result of Bland-Altman analysis between each pair of comparisons is shown in the Supporting Information.

Fig 5 shows four representative examples where the two-stage PET/LGE co-registration method apparently improved the co-alignment between the PET image and the LGE image. Lesions identified by LGE could have different FDG uptake values after co-registration. Bland-Altman plot of SUV mean $_{\text {changes }}$ of 40 LGE-enhanced lesions is shown in Fig 6. For fasting patients, the mean SUV in the lesion increased 
from $1.69 \pm 1.15$ to $1.84 \pm 1.16$ with only a trending significance ( $p$ value $=0.1260)$. For glucose loading patients, the mean SUV in the lesion significantly increased from $3.11 \pm 2.41$ to $3.67 \pm 2.68(p$ value $=0.0001$ ).

Histogram of the S-I direction displacement of LGE/PET is shown in Fig 7. Proportion of different displacements in the study population: 0 2 $\mathrm{mm}, 34.4 \%$; 0 4 $\mathrm{mm} \mathrm{50 \% ;} \mathrm{0} \mathrm{6} \mathrm{mm,} \mathrm{63.7 \% .}$

\section{Discussion}

The proposed two-stage registration method significantly improves the registration quality of PET/LGE in myocardial PET/MR imaging. The major finding from this study is of twofold. Firstly, the misregistration between PET and LGE is common with $50 \%$ of the studied population showing image mismatches along $\mathrm{S}$-I direction greater than $4 \mathrm{~mm}$. Secondly, the proposed co-registration method significantly improved the image quality compared with non-registration and one-stage registration, indicating that using the MRAC FLASH image as an intermedia to co-register PET and LGE is more effective than direct co-registration.

By offering more accurate SUV value, co-registration of PET and LGE may improve diagnostic accuracy in assessment of non-ischemic cardiomyopathy by integrated PET/MR. Eun-jung reported that ${ }^{18} \mathrm{~F}$-FDG myocardial PET not only shows fibrosis as late gadolinium enhancement (LGE) does on MRI but also its severity, which means the late stage of hypertrophic myocardium might show more decrease of ${ }^{18} \mathrm{~F}-\mathrm{FDG}$ uptake [15]. LGE can detects focal fibrosis in myocardium, it provides excellent risk stratification for sudden cardiac death, all-cause mortality, and heart failure hospitalization in patients with non-ischemic cardiomyopathy [16]. The combination of glucose loading ${ }^{18}$ F-FDG PET and LGE may provide solid evidence for the evaluation of myocardial vitality of DCM or burned-out HCM. Focal fibrosis by LGE in combination with focal myocardial inflammation by fasting PET additionally may suggest a poor prognosis for HCM. Since the accurate analysis of cardiomyopathy depends on the accurate matching of PET and LGE images, improved co-registration may lead to more accurate assessment of the diseased myocardium.

In cardiac PET/MR imaging, registration of cardiac images is a challenge because of the various sources and the specificity of each imaging modality. Fewer accurate anatomical landmarks and nonrigid moving making registration of cardiac images a complex [4]. Techniques developed for co-registration of PET and LGE in integrated PET/MR are rare, most of the study focus on co-registration of PET and MRAC. In 2019, Martin found that average respiratory misalignment of $(7 \pm 4) \mathrm{mm}$ of the PET images and the AC maps was observed in 18 (90\%) patients [17]. In 2021, Elia found that misalignment of MRAC and PET datasets commonly occurs in adenosine stress myocardial perfusion imaging (MPI) on a integrated PET/MR device. Manual adjustment was used to correct the misalignment between the PET and MRAC images [18]. Non-rigid registration of MRAC and PET was also used in MR-guided PET image reconstruction [19]. In two-stage registration, the single-phase PET that best matched to the Dixon 3D FLASH got essentially good attenuation correction (the registration score is $4.813 \pm 0.410$ ), and making 
quantitative analysis feasible. For the first time, PET/LGE co-registration is carried out on the basis of PET/MRAC accurate co-registration.

In our study, image co-registration of LGE/PET image2 was carried out by one-stage registration. However, once the uptake pattern of myocardium cannot show myocardial contour clearly (such as myocardium inflammation assessment), mismatches would be difficult to identify and correct. Since the MRAC FLASH image and the phase-resolved PET images were gated by the same bellows signal, the mismatch between FLASH and PET should be intrinsically small. The original cross-modality registration problem of 2D LGE and 3D PET images is then simplified into the intra-modality registration problem of 2D LGE and 3D Water-Dixon images. This somewhat explains why the qualitative comparison between one-stage and two-stage methods was still significant even in the fasting patient subgroup.

In subgroup analysis, the result from the glucose loading group was highly consistent with the comparison including all patients with higher FDG uptake in the myocardium than the blood. This is to be expected since all these patients in the subgroup had high myocardial FDG uptake. For the fasting group, the qualitative analysis still showed significant improvements of the two-stage registration method over the two other approaches, suggesting improved co-registration. However, the quantitative analysis using myocardial SUV as a surrogate showed only trending improvements. Nevertheless, the trending improvements of myocardial SUV were not surprising since these patients had a mixed pattern of SUV in the heart.

In Fig 6, we can see a better co-registration method could significantly change the SUV in the LGE lesion. In the glucose-loading group, the LGE-SUV was increased as a result of co-registration. This increase was because the LGE lesion before co-registration often co-aligned with the blood pool or the lung in the PET image. Although LGE may have lower FDG uptake than normal myocardium, the FDG uptake in the blood and lung was even lower. In the fasting group, the LGE-SUV was not significantly different with or without co-registration, since the FDG uptake pattern in this group was more diverse.

The method has several limitations. Firstly, the method assumes rigid motion along superior-inferior direction between the end-respiratory FLASH image and the LGE image. This assumption was based on the previous studies suggesting the heart displacement due to respiratory motion was greatest in the inferior-superior direction [20,21]. However, although the simplification of motion models renders the method more practical to use, inadequate co-registration may occur as a result of the simplification. Secondly, since the apex and fundus in the long-axis images often have inconsistent motion due to breathing, the method based on rigid motion correction is not suitable for long-axis MR images. Finally, the respiration-gated FLASH sequence used in this study was acquired without ECG-gating, resulting in blurred myocardium in some patients, which may lead to inaccurate registration.

In the assessment of cardiomyopathy, MR sequences like dark-blood T2-Weighted, LGE, Mapping are believed to play important role, and the mismatch between those sequence can also be corrected using the proposed two-stage registration. 


\section{Conclusion}

In conclusion, we have proposed a two-stage cardiac PET/LGE co-registration method for cardiac PET/MR imaging that is applicable for 2D short-axial LGE and 3D PET images. The technique may improve diagnostic accuracy of non-ischemic cardiomyopathies via better image co-registration. The technique provides a convenient and easy-to-operate solution to make myocardial PET/MR quantitative analysis feasible.

\section{Declarations}

Funding This work was supported by the National Natural Science Foundation of China (81871388, 62001288), Project of Science and Technology Commission of Shanghai Municipality (19DZ1930703), and Shanghai Pujiang Program (20PJ1408800).

Conflicts of interest/Competing interests All authors disclosed no relevant relationships.

Availability of data and material Not applicable

Code availability Not applicable

Authors' contributions Conceptualization: Zheng Zhang, Xing Chen, Jun Zhao, Chenxi Hu, Jianmin Yuan, Lingzhi Hu; Methodology: Zheng Zhang, Xing Chen, Qing Wan, Chenxi Hu, Zhe Wang; Formal analysis and investigation: Jun Zhao, Xing Chen, Qing Wan, Haiyan Wang, Na Qi,; Writing - original draft preparation: Zheng Zhang, Jianmin Yuan, Hongwei Sun, Zhe Wang; Writing -review and editing: Chenxi Hu, Jun Zhao, Jianmin Yuan, Lingzhi Hu, Hongwei Sun; Funding acquisition: Jun Zhao, Chenxi Hu; Resources: Jun Zhao, Chenxi Hu; Supervision: Jun Zhao, Lingzhi Hu.

Ethics approva/ Registered No. DF-2020-085, 2020.04.30

Consent to participate The data that support the findings of this study are available on request from the corresponding author. The data are not publicly available due to privacy or ethical restrictions.

Consent for publication Patients signed informed consent regarding publishing their data.

\section{References}

1. Nazir MS, Ismail TF, Reyes E, Chiribiri A, Kaufmann PA, Plein S. Hybrid positron emission tomographymagnetic resonance of the heart: current state of the art and future applications. Eur Heart $\mathrm{J}$ Cardiovasc Imaging. 2018;19:962-74. doi:10.1093/ehjci/jey090.

2. Jagsi R, Moran JM, Kessler ML, Marsh RB, Balter JM, Pierce LJ. Respiratory Motion of The Heart and Positional Reproducibility Under Active Breathing Control. International Journal of Radiation Oncology*Biology*Physics. 2007;68:253-8. doi:10.1016/j.ijrobp.2006.12.058. 
3. Nekolla SG, Martinez-Moeller A, Saraste A. PET and MRI in cardiac imaging: from validation studies to integrated applications. Eur J Nucl Med Mol Imaging. 2009;36 Suppl 1:S121-30. doi:10.1007/s00259008-0980-1.

4. Makela T, Clarysse P, Sipila O, Pauna N, Pham QC, Katila T, et al. A review of cardiac image registration methods. IEEE Trans Med Imaging. 2002;21:1011-21. doi:10.1109/TMI.2002.804441.

5. Le Meunier L, Maass-Moreno R, Carrasquillo JA, Dieckmann W, Bacharach SL. PET/CT imaging: Effect of respiratory motion on apparent myocardial uptake. Journal of Nuclear Cardiology. 2006;13:82130. doi:10.1016/j.nuclcard.2006.09.003.

6. Livieratos L, Rajappan K, Stegger L, Schafers K, Bailey DL, Camici PG. Respiratory gating of cardiac PET data in list-mode acquisition. Eur J Nucl Med Mol Imaging. 2006;33:584-8. doi:10.1007/s00259-0050031-0.

7. Ramalho M, AlObaidy M, Catalano OA, Guimaraes AR, Salvatore M, Semelka RC. MR-PET of the body: Early experience and insights. Eur J Radiol Open. 2014;1:28-39. doi:10.1016/j.ejro.2014.09.001.

8. Wells RG, Ruddy TD, DeKemp RA, DaSilva JN, Beanlands RS. Single-phase CT aligned to gated PET for respiratory motion correction in cardiac PET/CT. J Nucl Med. 2010;51:1182-90. doi:10.2967/jnumed.109.070011.

9. Lucignani G. Respiratory and cardiac motion correction with 4D PET imaging: shooting at moving targets. Eur J Nucl Med Mol Imaging. 2009;36:315-9. doi:10.1007/s00259-008-1017-5.

10. Camoni L, Santos A, Attard M, Mada MO, Pietrzak AK, Rac S, et al. Best practice for the nuclear medicine technologist in CT-based attenuation correction and calcium score for nuclear cardiology. Eur $\mathrm{J}$ Hybrid Imaging. 2020;4:11. doi:10.1186/s41824-020-00080-0.

11. Chen S, Gu Y, Yu H, Chen X, Cao T, Hu L, et al. NEMA NU2-2012 performance measurements of the United Imaging uPMR790: an integrated PET/MR system. Eur J Nucl Med Mol Imaging. 2021;48:1726-35. doi:10.1007/s00259-020-05135-9.

12. Peter L. Tilkemeier M, Jamieson Bourque, MD,Rami Doukky, MD, MSc, Rupa Sanghani, MD,and Richard L. Weinberg, MD, PhD. ASNC imaging guidelines for nuclear cardiology procedures, Standardized reporting of nuclear cardiology procedures. Journal of Nuclear Cardiology. 2017; Volume 24, Number 6;2064-128. doi:10.1007/s12350-017-1057-y.

13. Osborne MT, Hulten EA, Murthy VL, Skali H, Taqueti VR, Dorbala S, et al. Patient preparation for cardiac fluorine-18 fluorodeoxyglucose positron emission tomography imaging of inflammation. J Nucl Cardiol. 2017;24:86-99. doi:10.1007/s12350-016-0502-7.

14. Lee ES, Lee JM, Yu MH, Shin Cl, Woo HS, Joo I, et al. High spatial resolution, respiratory-gated, t1weighted magnetic resonance imaging of the liver and the biliary tract during the hepatobiliary phase of 
gadoxetic Acid-enhanced magnetic resonance imaging. J Comput Assist Tomogr. 2014;38:360-6. doi:10.1097/rct.0000000000000055.

15. Kong EJ, Lee SH, Cho IH. Myocardial Fibrosis in Hypertrophic Cardiomyopathy Demonstrated by Integrated Cardiac F-18 FDG PET/MR. Nucl Med Mol Imaging. 2013;47:196-200. doi:10.1007/s13139013-0201-0.

16. Yamaguchi H, Hasegawa S, Yoshioka J, Uehara T, Hashimoto K, Kusuoka H, et al. Characteristics of myocardial 18F-fluorodeoxyglucose positron emission computed tomography in dilated cardiomyopathy and ischemic cardiomyopathy. Ann Nucl Med. 2000;14:33-8. doi:10.1007/bf02990476.

17. Lassen ML, Rasul S, Beitzke D, Stelzmuller ME, Cal-Gonzalez J, Hacker M, et al. Assessment of attenuation correction for myocardial PET imaging using combined PET/MRI. J Nucl Cardiol. 2019;26:1107-18. doi:10.1007/s12350-017-1118-2.

18. von Felten E, Benetos G, Patriki D, Benz DC, Rampidis GP, Giannopoulos AA, et al. Myocardial creepinduced misalignment artifacts in PET/MR myocardial perfusion imaging. Eur $\mathrm{J}$ Nucl Med Mol Imaging. 2021;48:406-13. doi:10.1007/s00259-020-04956-y.

19. Munoz C, Ellis S, Nekolla SG, Kunze KP, Vitadello T, Neji R, et al. MR-guided motion-corrected PET image reconstruction for cardiac PET-MR. J Nucl Med. 2021. doi:10.2967/jnumed.120.254235.

20. Shechter G, Resar JR, McVeigh ER. Displacement and velocity of the coronary arteries: cardiac and respiratory motion. IEEE Trans Med Imaging. 2006;25:369-75. doi:10.1109/TMI.2005.862752.

21. Yoon JH, Lee JM, Lee ES, Baek J, Lee S, Iwadate Y, et al. Navigated three-dimensional T1-weighted gradient-echo sequence for gadoxetic acid liver magnetic resonance imaging in patients with limited breath-holding capacity. Abdominal Imaging. 2015;40:278-88. doi:10.1007/s00261-014-0214-x.

\section{Figures}




\section{A Two-Stage Method}

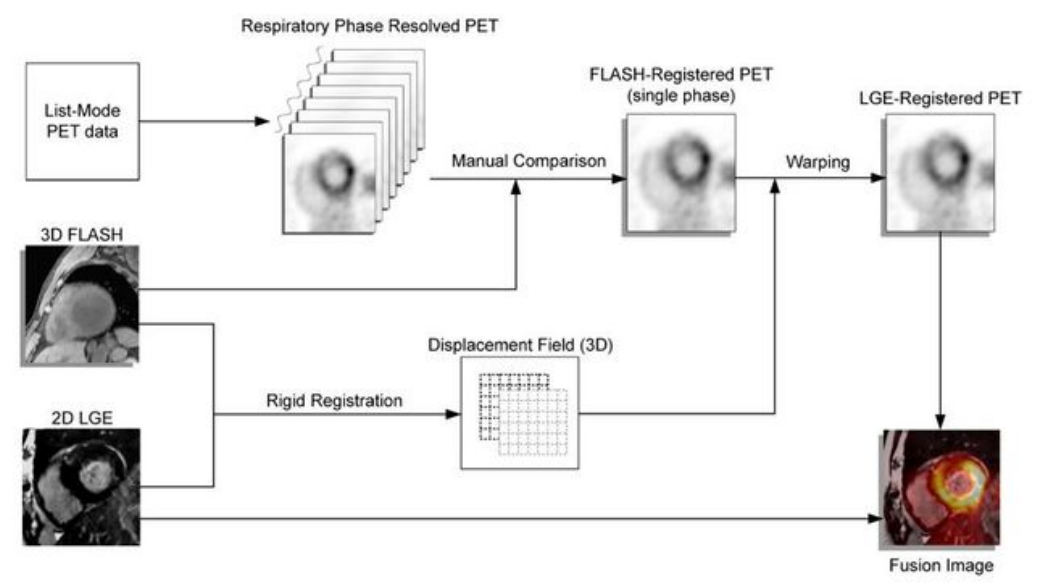

B One-Stage Method

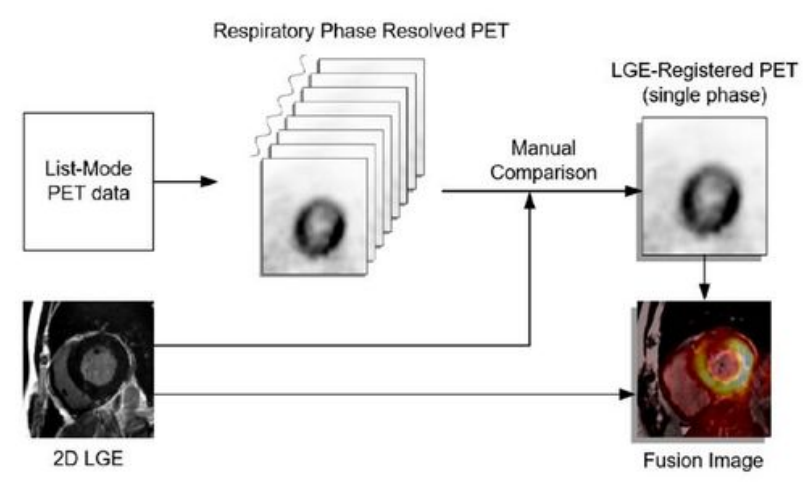

\section{Figure 1}

(A) Illustration of image co-registration of LGE and PET with two-stage registration. Firstly, List-Mode PET data was binned into eight respiratory bins based on respiratory signal. Secondly, compare FLASH and Respiratory Phase Resolved PET to choose one FLASH-Registered phase. Thirdly, perform rigid registration manually between 3D FLASH and 2D LGE and get the 3D displacement field. Finally, wraps FLASH-Registered PET use the input displacement field to generate LGE-Registered PET. (B) Illustration of image co-registration of LGE and PET with one-stage registration. Firstly, List-Mode PET data was binned into eight respiratory bins based on respiratory signal. And then, compare 2D LGE and Respiratory Phase Resolved (RPR) PET to choose one LGE-Registered phase. 


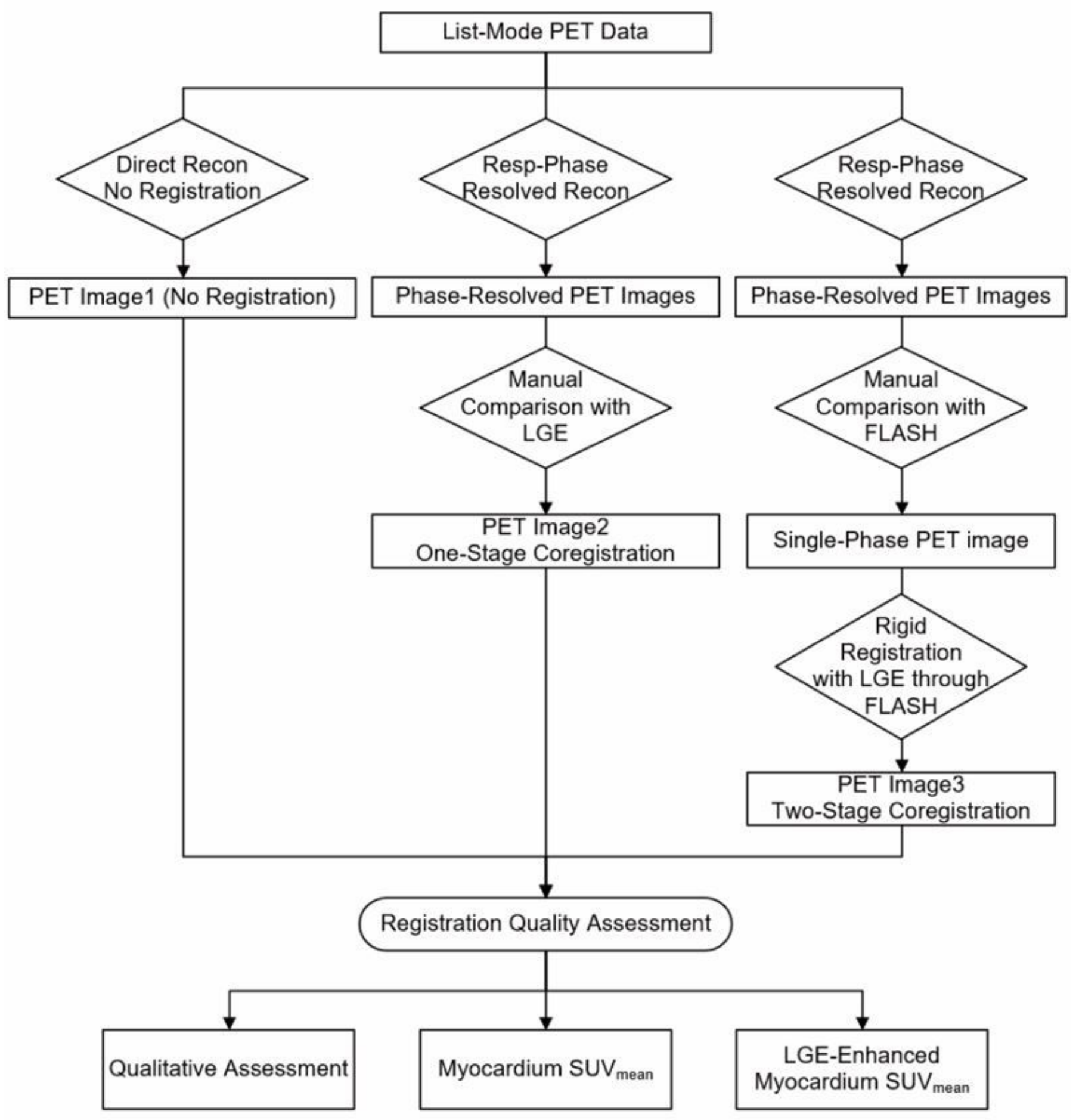

Figure 2

Schematic layout of the study. 

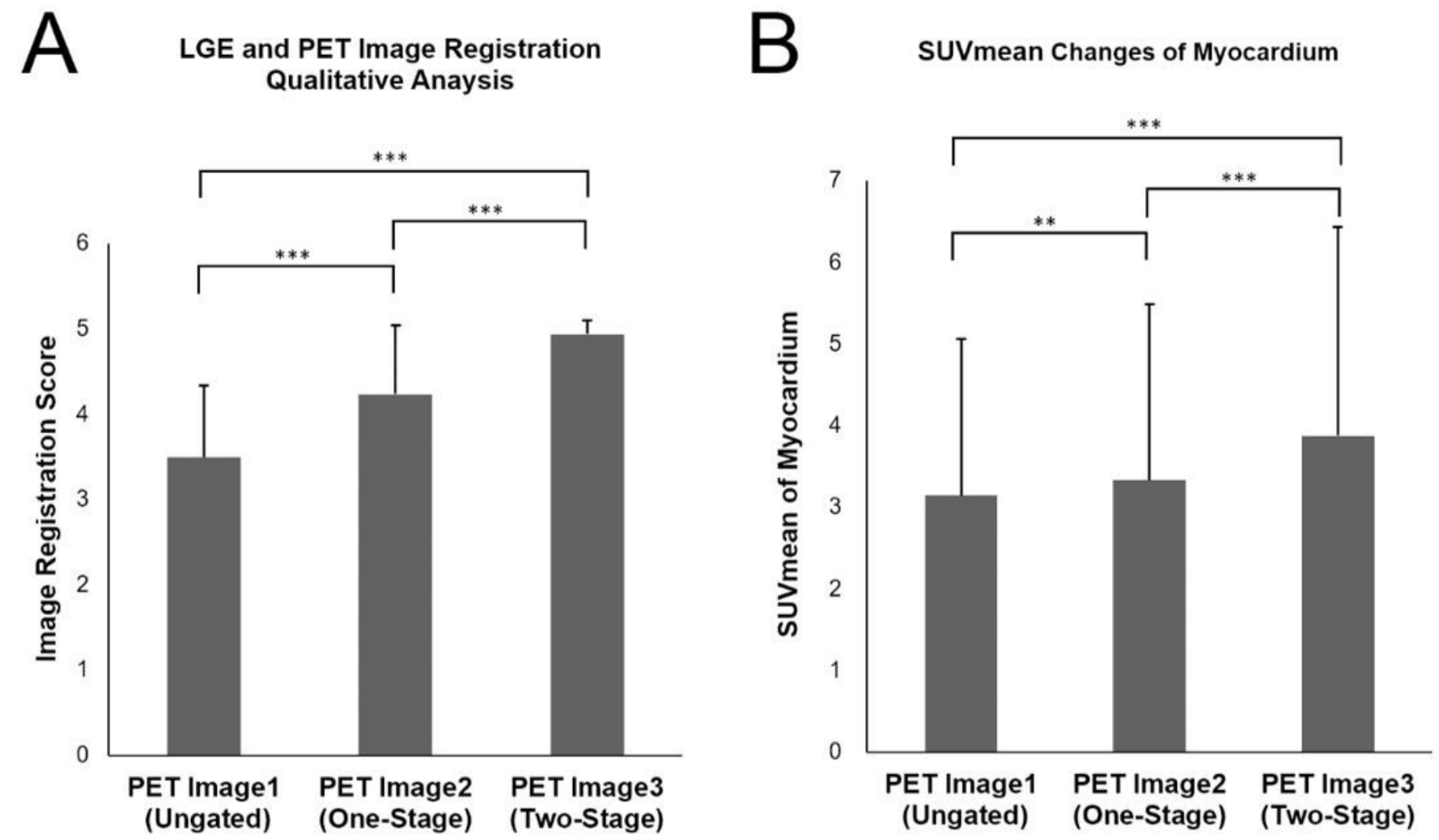

\section{Figure 3}

Registration quality assessment of PET image. (A) Image registration score of PET image1/2/3. PET image3 have the best registration accuracy and the increase in registration accuracy is statistically significant. (B) SUVmean of myocardium of PET image1/2/3. The increase in SUVmean of myocardium is statistically significant. Note: ${ }^{*}{ }^{\star *},{ }^{\star \star \star *}$ represent $p$ value $<0.05,0.01$ and 0.001 respectively.
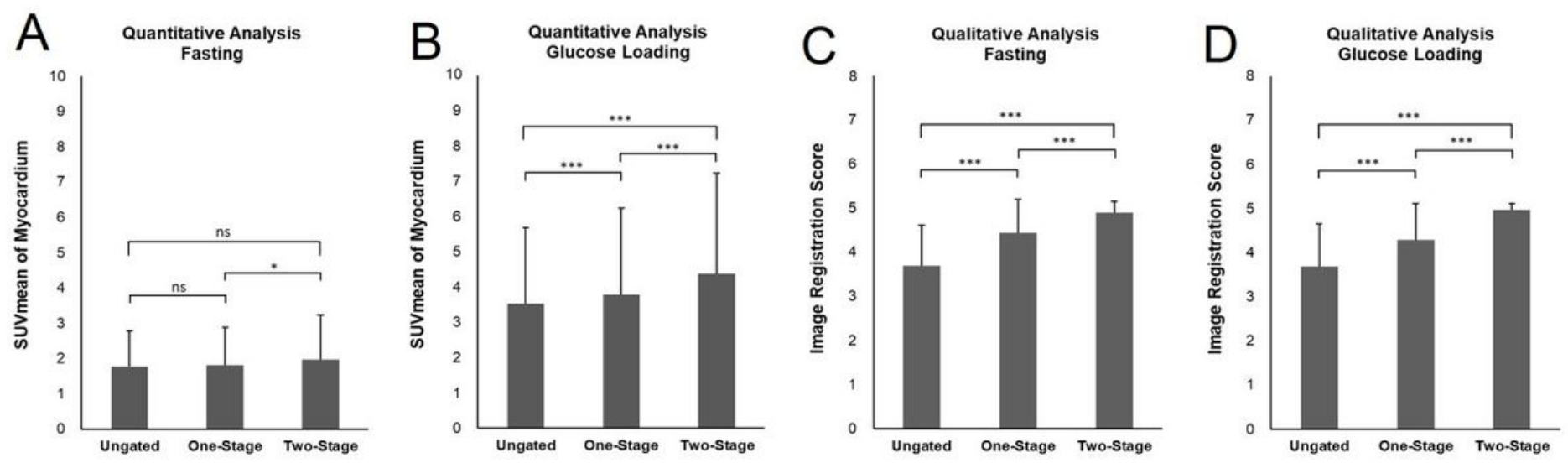

Figure 4 
Registration quality quantitative and qualitative subgroup analysis. SUVmean of myocardium of PET images (Ungated, One-Stage and Two-Stage) of fasting showed Two-Stage method only made trending improvements. The $p$ value between Two-Stage and One-Stage Images is 0.033 , Two-Stage and Ungated Images is $0.056(\mathrm{~A})$. The increase in SUVmean of myocardium is statistically significant for glucose loading (B). For qualitative analysis, Two-Stage PET image have the best registration accuracy and the increase in registration accuracy is statistically significant $(C, D)$. Note: ns, $*, * \star, * \star *$ represent Not Statistically Significant, $p$ value $<0.05,0.01$ and 0.001 respectively. 

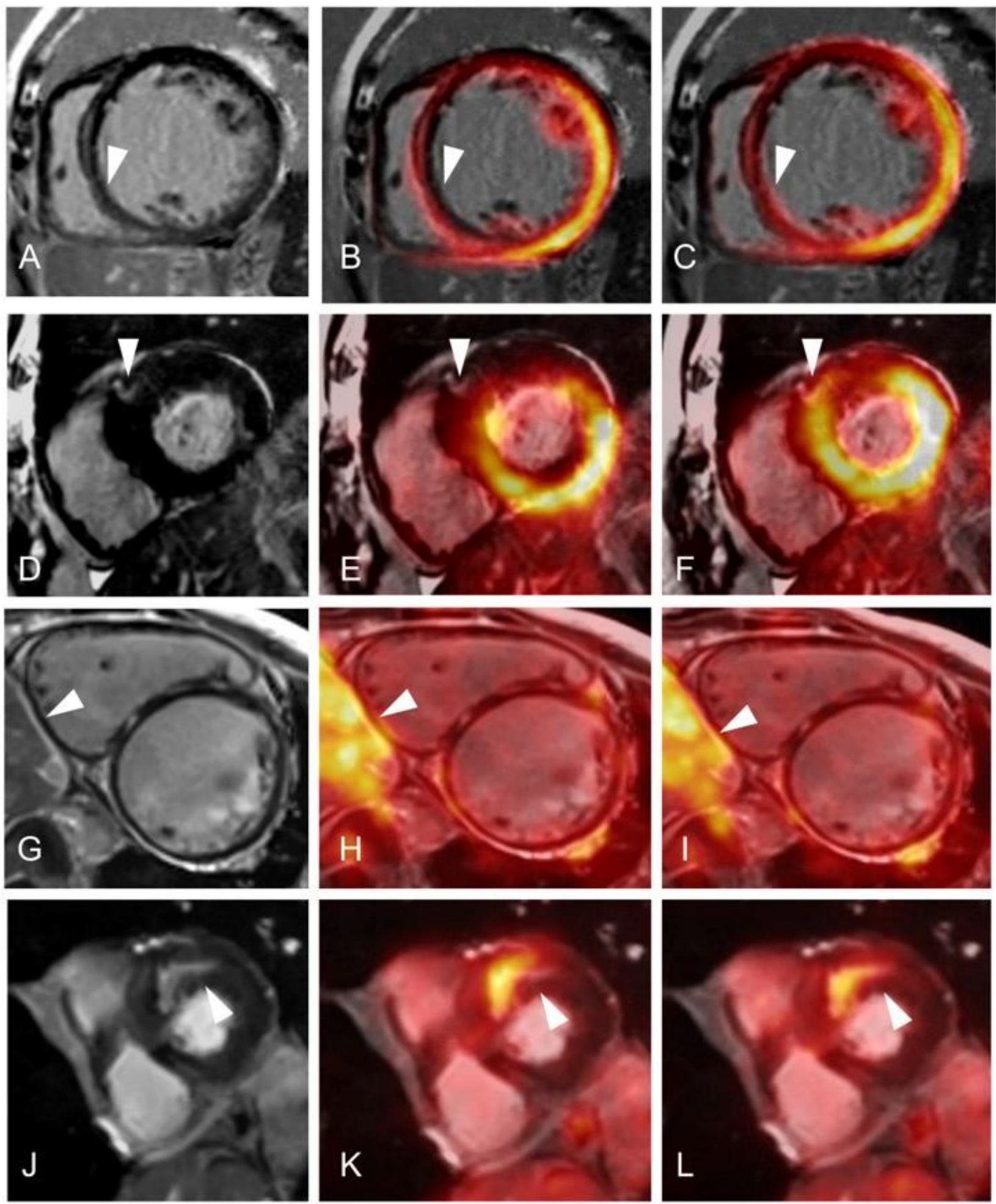

\section{Figure 5}

Comparison of images before and after two-stage registration. (A) Short-axial LGE image of one patient with DCM underwent fasting PET imaging. (B) LGE and PET fusion image before registration, obvious mismatch between LGE and PET can be seen (arrow). (C) After two-stage registration, displacement between LGE and PET can hardly be detected. (D-F) SUVmean of one LGE-enhanced lesion changed after two-stage registration for one patient with HCM underwent glucose loading PET imaging (arrow). (G-I) For 
one patient with DCM underwent glucose loading PET imaging, myocardium have a low FDG uptake pattern, but the elimination of displacement of liver can be seen after two-stage registration (arrow). (J-L) The enhanced pattern in LGE image match exactly with FDG uptake pattern after two-stage registration for one patient with HCM underwent fasting PET imaging (arrow).
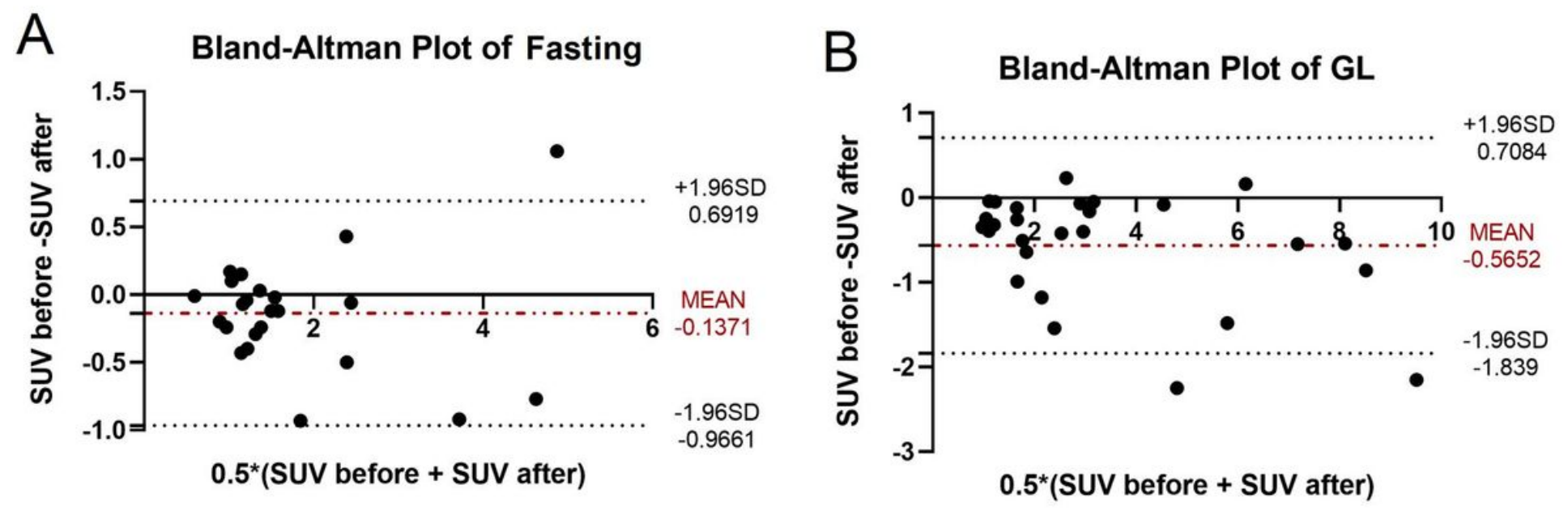

\section{Figure 6}

Bland-Altman plot of SUVmean change of LGE-Enhanced lesion after two-stage registration of fasting (A) and glucose loading (B) imaging preparation. 


\section{Histogram of Displacement}

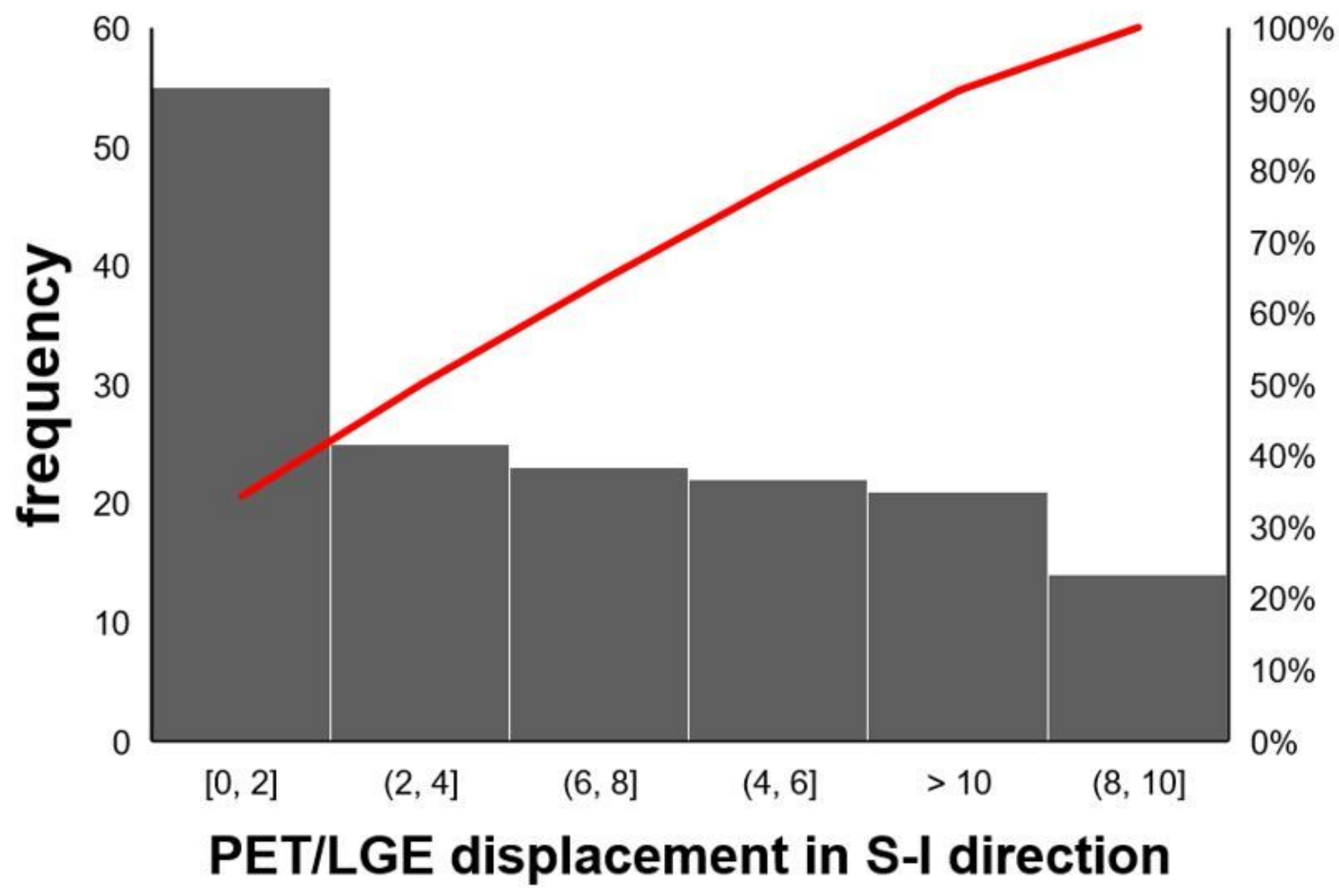

Figure 7

Histogram of PET/LGE diaplacement in S-I direction.

\section{Supplementary Files}

This is a list of supplementary files associated with this preprint. Click to download.

- S1.pdf 